\title{
MODELAGEM DE USINAS EÓLICAS PARA ESTUDOS DE CONFIABILIDADE
}

\author{
Andréa P. Leite* \\ Carmen L.T. Borges ${ }^{\dagger}$ \\ Djalma M. Falcão ${ }^{\dagger}$ \\ *Eletrobrás - Centrais Elétricas Brasileiras S.A. \\ DETE - Divisão de Estudos Elétricos \\ Rio de Janeiro - RJ \\ ${ }^{\dagger}$ COPPE/UFRJ - Programa de Engenharia Elétrica \\ Caixa Postal 68504 \\ CEP 21941-972 - Rio de Janeiro - RJ
}

\section{RESUMO}

Este trabalho desenvolve um modelo computacional de representação probabilística da geração de usinas eólicas para estudos de confiabilidade. O modelo pode fornecer a estimativa anual da energia produzida e calcular indicadores de desempenho, que podem ser usados na análise de viabilidade de implantação das usinas. O modelo combina as características aleatórias da velocidade do vento às informações operativas das turbinas, tais como as taxas de falha e de reparo, representando o comportamento da usina eólica por um processo de Markov. Foram feitas simulações com séries reais de velocidade de vento de regiões do Brasil e os resultados reproduziram com sucesso o comportamento de todos os componentes presentes no modelo.

PALAVRAS-CHAVE: Confiabilidade de Sistemas de Potência, Geração Eólica, Modelo de Usinas Eólicas.

\footnotetext{
Artigo submetido em 06/06/2005

1a. Revisão em 10/10/2005

2a. Revisão em 17/05/2006

Aceito sob recomendação do Editor Associado Prof. Carlos A. Castro
}

\begin{abstract}
This paper develops a computer model for the probabilistic representation of wind farms generation for reliability studies. The model can provide an annual estimation of energy production and calculate performance indices, which can be used on wind farms implantation feasibility analysis. The model combines the stochastic characteristics of wind velocity with the operational information of the turbines, such as the failure and repair rates, representing the wind farm by a Markov process. The simulations were made with real series of wind velocity of several Brazilian regions and the results reproduced successfully the behavior of the components considered in the model.
\end{abstract}

KEYWORDS: Power Systems Reliability, Wind Generation, Wind Farm Modeling.

\section{INTRODUÇÃO}

Nos últimos anos tem ocorrido um aumento do interesse na utilização de fontes renováveis para a geração de energia elétrica. Dentre as razões para esse interesse, pode-se destacar a necessidade de se buscar alternativas que ajudem a reduzir o uso de combustíveis fósseis devido ao seu alto custo. 
Além disso, existe o objetivo mundial da redução de emissões de gases poluentes na atmosfera, firmado pelo Protocolo de Kyoto em 1997, no Japão.

A energia eólica é, sem dúvida, uma das fontes alternativas de energia com exploração mais bem sucedida atualmente. Uma razão para este fato é a política de incentivo feita por vários países, assegurando a compra da energia eólica produzida, ainda que ela não ofereça preços competitivos. A Alemanha e a Dinamarca foram pioneiras neste procedimento, seguido por vários países, inclusive pelo Brasil, com a criação do PROINFA. Além disso, pode-se destacar o avanço tecnológico, tanto em pesquisa quanto em desenvolvimento, fazendo com que ocorra uma rápida redução no custo de utilização desta forma de geração.

No entanto, a geração eólica possui desvantagens como fonte regular de energia, e por isso é considerada menos confiável que as fontes convencionais. A quantidade de energia diária disponível pode variar muito de uma estação do ano para outra, em um mesmo local, e seu uso fica limitado a lugares de ventos fortes e relativamente constantes. Empresas que necessitam avaliar a implantação deste tipo de geração preocupam-se sobretudo com a intermitência do vento e com o que isto pode ocasionar no planejamento e na operação de seus sistemas elétricos. A conexão de um número cada vez maior de usinas eólicas aos sistemas elétricos implica na necessidade de se estudar os seus efeitos.

As características operativas da usina eólica, fortemente dependentes do regime local dos ventos, fazem com que o modelo de geração de uma usina convencional não possa ser diretamente aplicado a ela. Neste trabalho, é proposto um modelo probabilístico, que pode ser usado em estudos de confiabilidade, bem como no planejamento de sistemas em que se deseja avaliar a viabilidade de implantação da geração eólica.

Alguns trabalhos sobre confiabilidade de fontes não convencionais de energia, incluindo usinas eólicas, já foram desenvolvidos. O primeiro a se destacar foi (Giorsetto e Utsurogi, 1983), que introduziu o conceito da intermitência da geração e das taxas de falha e reparo das turbinas eólicas. Em (Singh e Kim, 988) este conceito foi combinado com a divisão do sistema elétrico em subsistemas de geração, separando as fontes convencionais das não convencionais, onde os estados de geração são tratados como variáveis aleatórias. Em (Billinton et alii, 1996), as características cronológicas da velocidade do vento foram consideradas na avaliação da confiabilidade de sistemas, utilizando-se simulação Monte Carlo. Em (Billinton e Karki, 2001) foi avaliada a confiabilidade de sistemas isolados em que se deseja implantar a geração não convencional, utilizando-se os conceitos mencionados acima. Em (Sayas e Allan, 1996), a disponibilidade de geração de uma usina eólica foi calculada utilizando-se um modelo probabilístico, o qual foi utilizado como ponto de partida para o desenvolvimento do presente trabalho.

\section{GERAÇÃo EÓLICA}

A geração de energia elétrica a partir da energia contida nos ventos é realizada com o uso de turbinas eólicas. A turbina eólica é constituída por uma série de componentes, responsáveis pela produção da energia elétrica em três etapas: a captação da energia cinética contida no vento, a conversão da energia cinética em energia mecânica e a transformação da energia mecânica em energia elétrica propriamente dita, pelo gerador elétrico, ao final do processo.

De um modo geral, os fatores que interferem na velocidade do vento e, consequentemente, na geração, são:

- Condições Climáticas: variações de temperatura, regime de ventos, monções, etc.

- Características do Local: rugosidade do terreno, orografia, presença de obstáculos, etc.

\subsection{Potência Extraída do Vento}

A potência extraída pela turbina eólica de um fluxo de ar de densidade $\rho$, movendo-se a uma velocidade $v$, perpendicular a uma seção transversal de área $A$ de um cilindro varrido pelas hélices da turbina, pode ser expressa como:

$$
P=\frac{1}{2} C_{p} \rho A v^{3}
$$

onde $C p$ é o coeficiente de potência da turbina. Este coeficiente representa a eficiência aerodinâmica da turbina e mostra quanto de energia do vento a turbina é capaz de converter em potência mecânica, para cada velocidade de vento. Como a geração é proporcional ao cubo da velocidade do vento, as variações de geração de potência podem ser muito grandes.

A fim de permitir algum controle da geração, são introduzidos os controles de estol e de passo. Velocidades muito altas podem provocar danos nas pás e nos outros componentes da turbina. Por essa razão, o dispositivo de controle limita o funcionamento da turbina até uma velocidade de corte, mais comumente conhecida como cut out. Esta velocidade está em torno de 20 a $25 \mathrm{~m} / \mathrm{s}$. Por outro lado, velocidades baixas fazem a turbina gerar potências muito baixas e são insuficientes para a partida do gerador. A velocidade inicial de funcionamento da turbina, conhecida como velocidade de acionamento ou cut in, está em torno de 3 a $5 \mathrm{~m} / \mathrm{s}$. Dessa forma, a operação da turbina fica limitada ao intervalo entre 


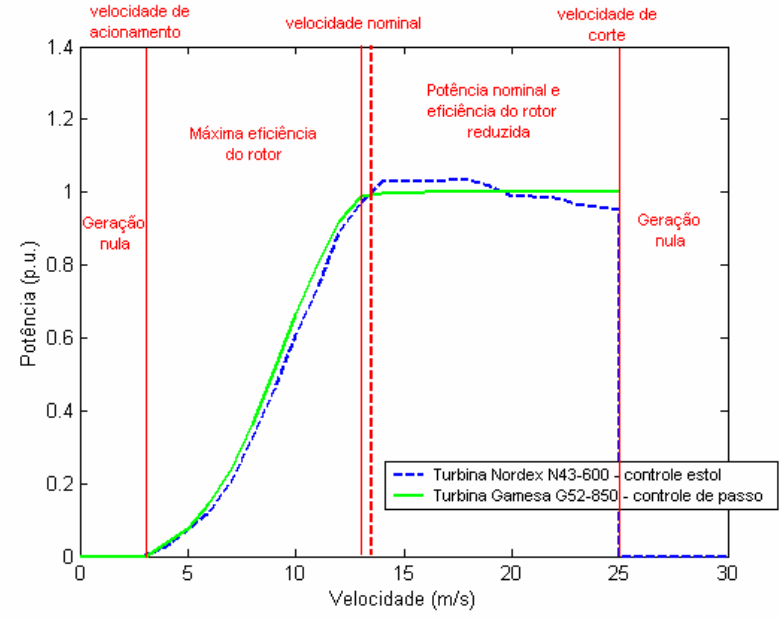

Figura 1: Curvas de potência de turbinas - controles distintos

a velocidade de acionamento e a velocidade de corte. Para valores fora desse intervalo, a potência gerada é nula.

A curva de potência de uma turbina eólica representa a sua característica de operação $P(v)$. A figura 1 mostra a curva de potência de duas turbinas comerciais, com controles tipo passo e estol. Observa-se que para controle do tipo passo, a geração permanece constante e com o valor igual à potência nominal entre $v_{\text {nom }}$ e $v_{\text {cut-out }}$. Já para controle do tipo estol, há uma variação da geração em torno do valor nominal.

Pode-se observar na curva de potência que existe uma variação de potência bem maior nos períodos de ventos leves e moderados, que ocorrem com maior freqüência. Esta característica acentua ainda mais a necessidade de se incluir a influência da velocidade do vento no modelo de confiabilidade da usina eólica.

\subsection{Comportamento Aleatório do Vento}

As variações que o vento pode apresentar são definidas como variações de curta duração, diárias, sazonais e anuais. As variações de curta duração são aquelas que ocorrem em intervalos de segundos, tais como as turbulências e as rajadas de vento. Elas não são utilizadas em estudos de viabilidade de implantação, nem em estudos de confiabilidade e estimativa de geração de um sítio, a sua relevância está associada aos estudos estruturais, bem como às variações de tensão e frequiência em estudos de estabilidade.

As variações diárias e sazonais são importantes para uma estimativa do suprimento da demanda de um sistema elétrico. Por exemplo, sabendo-se que na maioria das vezes a velocidade do vento é maior durante o dia que durante a noite, se o pico de carga do sistema ocorrer durante o dia, ele pode ser suprido tanto pelas usinas convencionais, desempenhando o papel de geração de base, quanto pelas usinas eólicas existentes, desempenhando o papel de geração de ponta.

A variação anual de velocidade de vento é importante para estudos de viabilidade de implantação de usinas eólicas e estudos de confiabilidade, porque fornece um conhecimento do regime local dos ventos, sendo a variação neste caso bem menor do que a variação sazonal. Para a análise do comportamento do vento em um determinado sítio, é necessário o conhecimento de registros de velocidade de vento durante longos períodos de tempo. Como estes registros são feitos em intervalos de tempo pequenos, o volume de dados é muito grande, tornando difícil o manuseio. Assim, é comum o agrupamento dos dados para permitir o levantamento da distribuição de frequiência de ocorrência de velocidades, conforme será visto mais adiante.

\section{MODELO DA USINA EÓLICA}

O modelo de confiabilidade deste trabalho procura considerar todos os fatores que interferem na geração de uma usina eólica, modelando o comportamento estocástico da velocidade do vento, assim como o comportamento operativo das turbinas, descrito pelas taxas de falha e de reparo, pela curva de potência, etc. Os resultados obtidos podem ser facilmente integrados no modelo do sistema no qual deseja-se avaliar a confiabilidade.

\subsection{Modelo da Turbina Eólica}

O modelo da turbina eólica deve representar todas as suas possibilidades operativas e a possibilidade de saída de operação por paradas programadas para manutenção e saídas forçadas. Cada turbina deve ser representada como um componente com taxas de falha e de reparo e com diferentes estados de geração.

A taxa de falha de uma turbina pode ser calculada a partir do seu histórico operativo como:

$$
\lambda=\frac{N_{f}}{h_{S}} \times 8760
$$

onde $\lambda$ é a taxa de falha da turbina ou taxa de transição do estado operativo para o estado falho, em ocorrências por ano, $N_{f}$ é o número de desligamentos forçados ocorridos e $h_{s}$ é o número de horas em serviço da turbina.

O tempo médio de reparo $(m)$ da turbina é uma função das condições climáticas, da parte da turbina afetada, da logística de operação e manutenção da usina e da velocidade do vento no instante da falha. A taxa de reparo é definida como $\mu=$ 

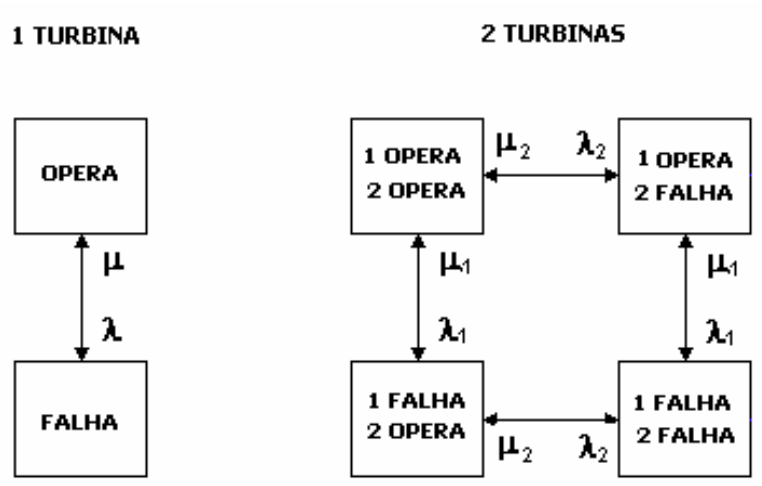

Figura 2: Diagramas de estados para uma e duas turbinas

$1 / m$.

A turbina eólica é modelada, neste trabalho, como um componente a dois estados: em operação e em falha. Quando ela está em estado operativo, a geração é determinada pela velocidade do vento e por sua característica $P(v)$, conforme apresentado na seção 2.1. A transição do estado operativo para o estado de falha é caracterizada pela taxa de falha $\lambda$ e a transição do estado de falha para o estado operativo é caracterizada pela taxa de reparo $\mu$.

Para uma usina com $\mathrm{N}$ turbinas eólicas, o número de possíveis estados operativos passa para $2^{N}$. Na figura 2 está a representação dos diagramas de espaço de estados para uma e duas turbinas diferentes, com taxas de falha $\lambda_{1}$ e $\lambda_{2}$ e de reparo $\mu_{1}$ e $\mu_{2}$, respectivamente.

\subsection{Modelo do Comportamento do Vento}

O comportamento do vento é modelado como um processo estocástico, onde a variável aleatória é a velocidade do vento e o índice do processo é o tempo. O diagrama esquemático do comportamento do vento utilizado no modelo, construído conforme a cadeia de Markov, está representado na figura 3.

Os estados de vento estão representados em ordem crescente de velocidade e a transição do estado $j-1$ para o estado $j$ é quantificada pela taxa $\lambda_{j-1, j}$. Conforme pode ser visualizado, o modelo permite a transição entre estados não adjacentes, permitindo a representação de grandes variações de velocidade em pequenos instantes.

Para que um sistema possa ser representado por uma cadeia de Markov, é necessário que a ocorrência de um estado qualquer dependa apenas da ocorrência do estado anterior. Além disso, o processo precisa ser estacionário, ou seja, com comportamento constante durante todo o tempo, independentemente do ponto de partida escolhido. Isto implica também

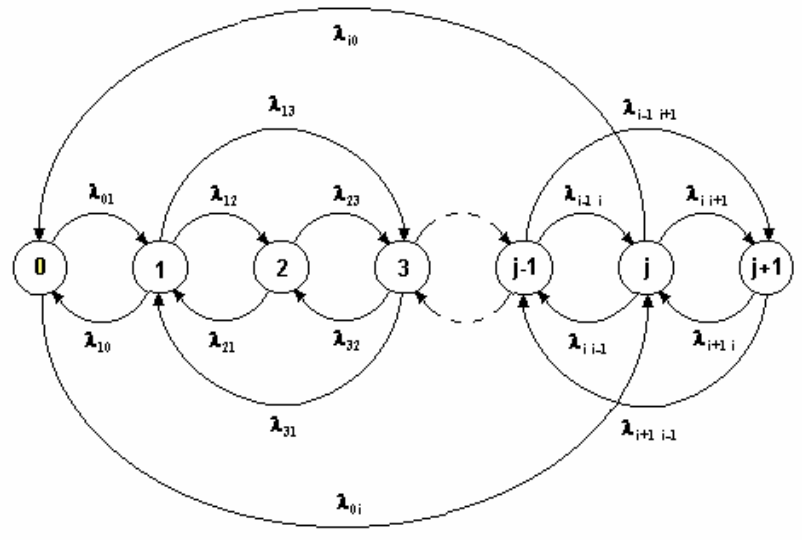

Figura 3: Cadeia de Markov para representação do vento

em afirmar que as taxas de transição entre estados são constantes durante todo o processo. Uma das características de um processo estacionário é que seu valor médio e seu desvio padrão são constantes, independentemente do ponto de partida dos dados analisados. Devido às variações sazonais do vento, a velocidade média e o desvio padrão não são constantes ao longo de um período, que pode ser um dia, um mês ou até mesmo uma estação do ano. Portanto, a rigor, o vento não é um processo estacionário (Corotis et alii, 1978). Entretanto, este efeito pode ser desprezado se as medições em estudo não seguirem uma tendência específica de um período qualquer ou a quantidade de dados for suficientemente grande, englobando um grande período de tempo, como um ano ou vários anos (Thomann e Barfield, 1998).

O comportamento de um processo estacionário sugere o uso de uma distribuição exponencial para a representação do tempo de permanência em um determinado estado. Esta distribuição é caracterizada por uma taxa de transição constante, definida pela expressão:

$$
\lambda_{i j}=\frac{N_{i j}}{D_{i}}
$$

onde $\lambda_{i j}$ é a taxa de transição do estado $i$ para o estado $j$, $N_{i j}$ é o número de transições entre o estado $i$ e o estado $j$ e $D_{i}$ é o tempo de permanência ou duração no estado $i$, dada por:

$$
D_{i}=\sum_{i=1}^{n} t_{i}
$$

ou seja, é o somatório dos $n$ intervalos de tempo em que o estado $i$ ocorreu. A probabilidade de ocorrência do estado $i$, observado $n$ vezes nas medições, é dada por: 


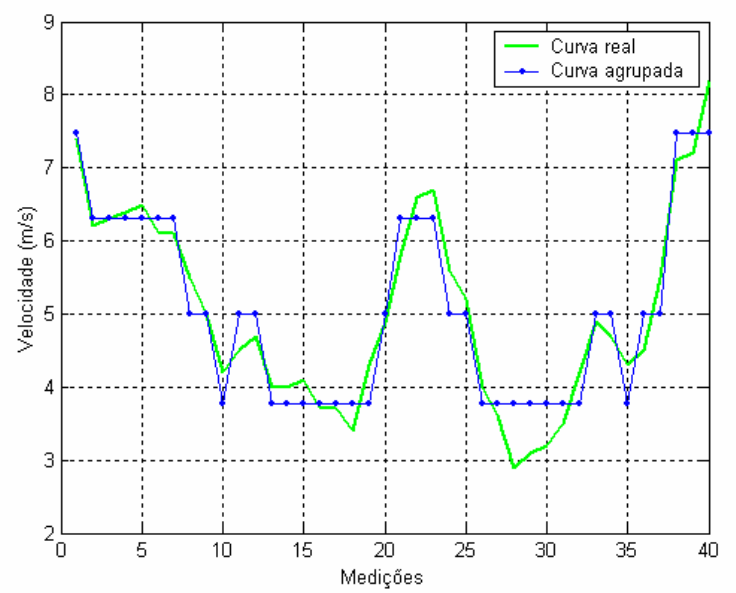

Figura 4: Trecho de série temporal de velocidade de vento e representação do agrupamento em 4 estados

$$
P_{i}=\frac{D_{i}}{T_{S}}
$$

onde $T_{s}$ é o tempo total da série temporal de velocidade de vento. A freqüência de ocorrência $F_{i}$ do estado $i$ é dada pelo somatório do número de ocorrências deste estado.

\subsubsection{Agrupamento Estatístico das Velocidades}

Devido ao grande número de estados de velocidade de vento presente em uma série temporal anual, a representação de todos eles em um modelo pode se tornar inviável. Por essa razão, decidiu-se por adotar uma técnica de agrupamento destas velocidades em um número menor de estados.

A finalidade de uma técnica de agrupamento é a alocação de elementos em grupos que representem alguma característica em comum. Em geral, as técnicas de agrupamento são de natureza iterativa e testam diversas alternativas de combinação de grupos, até que a função objetivo implícita seja minimizada. Para isso, são necessárias, primeiramente, a identificação do número de grupos que se deseja utilizar e as medidas que quantifiquem a similaridade de um elemento com um grupo. O algoritmo utilizado neste trabalho é baseado em uma técnica estatística, conhecida como K-means (MacQueen, 1967; Puntar, 2003). A figura 4 mostra a curva real de um trecho com 40 medições da série de um sítio estudado e a curva formada pelo agrupamento em 4 estados.

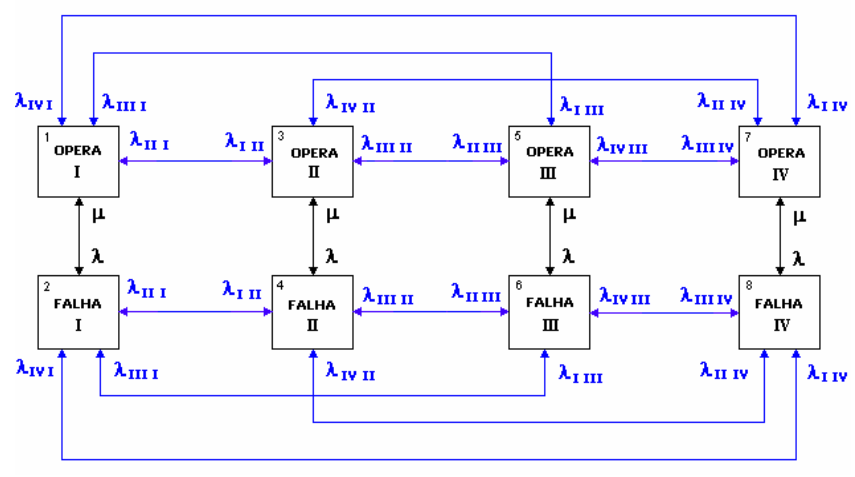

Figura 5: Diagrama de estados de uma usina eólica

\subsection{Modelo da Usina Eólica: Combinação dos Modelos da Turbina e do Vento}

A combinação dos estados operativos de uma turbina com o comportamento do vento pode ser representada em um diagrama de espaço de estados, conforme a figura 5. As transições entre o estado de operação e falha da turbina estão representadas pelas taxas de falha e reparo $\lambda$ e $\mu$, respectivamente. A velocidade do vento está agrupada nos estados I, II, III e IV e as transições entre eles estão representadas pelas taxas $\lambda_{i j}$, onde $\mathrm{i}$ representa o estado de vento inicial e $\mathrm{j}$ o estado final. Foram criados 8 estados, numerados no canto esquerdo superior. Para $N$ turbinas e $n$ estados de velocidade de vento, o número de estados resultante é dado pela expressão:

Estados da usina $=2^{N} \times n$

É importante lembrar que a transição entre estados de vento ocorre independentemente da transição entre estados de turbina e, em cada instante de tempo, apenas uma destas transições pode ocorrer. Se esta premissa não for estabelecida, a usina não pode ser modelada por um processo de Markov.

Para mais de uma turbina, o diagrama de espaço de estados transforma-se em um diagrama de múltiplas camadas, onde cada uma delas representa um estado de velocidade de vento e possui a representação de todos os estados operativos das turbinas. Na figura 6 está representado um trecho do diagrama de uma usina contendo duas turbinas.

\subsection{Metodologia de Cálculo}

As probabilidades dos estados de geração de uma usina eólica são encontradas através da solução do sistema estocástico: 


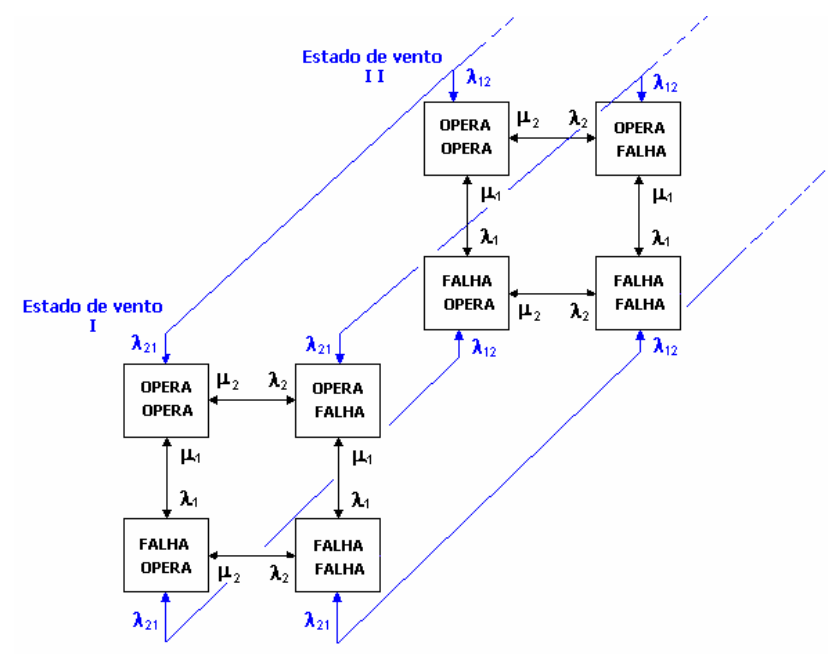

Figura 6: Diagrama de estados de usina eólica com 2 turbinas

$$
\underline{\dot{p}}(t)=\underline{p}(t) A
$$

onde $A$ é a matriz de intensidade de transição, cuja soma dos elementos de uma linha é sempre igual a zero. O objetivo do modelo é encontrar as probabilidades em regime permanente, também conhecidas como probabilidades estacionárias dos estados de geração da usina eólica, que são calculadas pela solução do sistema:

$$
\underline{p}(t) A=0
$$

Dessa forma, a solução do sistema permite encontrar as probabilidades dos estados de operação da usina. Cada estado representa um valor de geração da usina, definido através da condição operativa da turbina e do estado de velocidade de vento.

Entretanto, quando existem mais de uma turbina na usina, alguns estados operativos fornecem a mesma geração de saída. Estes estados podem ser agrupados através da soma das linhas que os representam na matriz A. Este procedimento é importante na modelagem de usinas que possuem muitas turbinas e/ou muitos estados de velocidade de vento, porque contribui para a redução da dimensão da matriz A.

Considerando-se que todas as turbinas são idênticas, ou seja, possuem a mesma característica de potência, a geração de cada estado da usina é determinada através da curva de potência, conforme explicado na seção 2.1. Dessa forma, a potência $P_{i}$ de um estado $i$, cuja velocidade é $v_{i}$, é dada por:

$P_{i}=$ Número de turbinas em operação no estado $t \times P\left(v_{i}\right)$ onde $P\left(v_{i}\right)$ é a potência gerada pela turbina no estado de vento $i$, de acordo com sua curva de potência. Para turbinas diferentes, deve ser obtido o valor da potência gerada a partir da curva de potência $P\left(v_{i}\right)$ de cada turbina e estes valores são somados para se obter a geração da usina.

Com as probabilidades dos estados de geração, pode-se montar a curva de distribuição de probabilidade acumulada, conforme mostra a figura 7. Ela representa a probabilidade da potência de geração da usina ser menor do que um valor $P_{i}$ qualquer. Esta curva pode ser utilizada no sorteio da probabilidade de operação da usina, em estudos de confiabilidade probabilística, que utilizem simulação Monte Carlo, por exemplo. Outra curva muito utilizada é a curva de duração, conforme mostra a figura 8 , que representa a porcentagem do tempo de permanência nos diferentes estados de geração. Esta curva é geralmente utilizada na estimativa da geração da usina e em estudos de viabilidade de implantação de usinas eólicas.

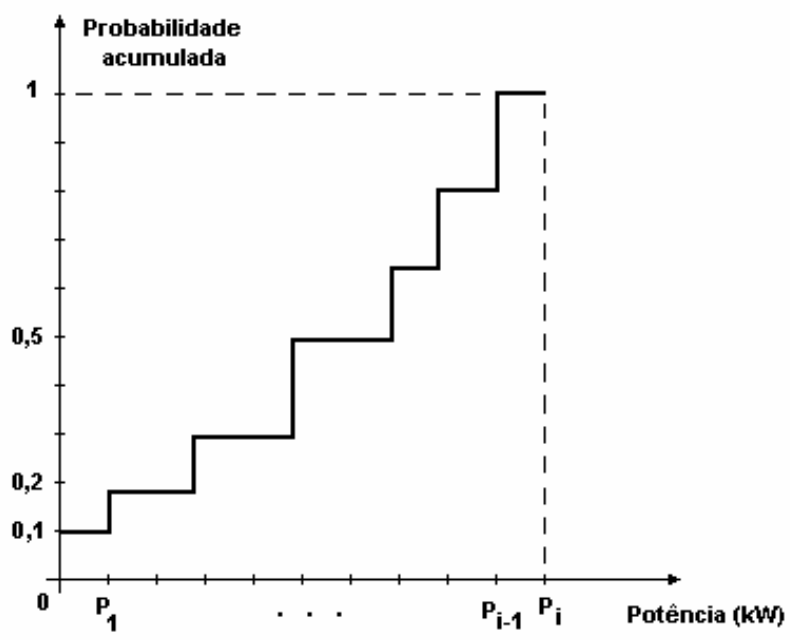

Figura 7: Curva de distribuição de probabilidade acumulada

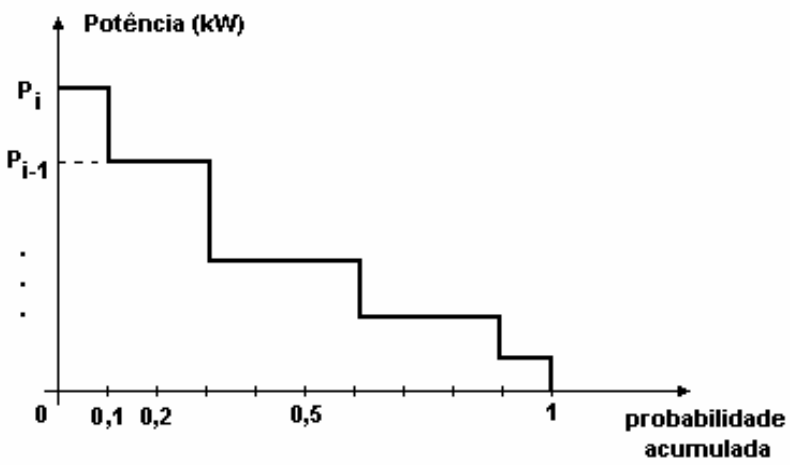

Figura 8: Curva de duração de uma usina eólica 


\begin{tabular}{|l|c|c|c|}
\cline { 2 - 4 } \multicolumn{1}{c|}{} & Nordeste & Sul & Sudeste \\
\hline Número de estados diferentes & 165 & 196 & 179 \\
\hline Média (m/s) & 7,58 & 7,00 & 6,59 \\
\hline Valor mínimo (m/s) & 0 & 0 & 0 \\
\hline Valor máximo (m/s) & 18,7 & 20 & 18,7 \\
\hline
\end{tabular}

Tabela 1: Dados de vento dos sítios estudados

\begin{tabular}{|l|c|}
\hline Potência nominal $(\mathrm{kW})$ & 1500 \\
\hline Velocidade nominal $(\mathrm{m} / \mathrm{s})$ & 12 \\
\hline Velocidade de acionamento $(\mathrm{m} / \mathrm{s})$ & 3 \\
\hline Velocidade de corte $(\mathrm{m} / \mathrm{s})$ & 20 \\
\hline Tipo de controle & pitch \\
\hline
\end{tabular}

Tabela 2: Dados técnicos da Turbina A

\section{RESULTADOS}

\subsection{Dados Utilizados}

Para o teste do modelo foram utilizadas três séries de vento reais de regiões do Brasil: uma no Nordeste, uma no Sul e uma no Sudeste. O intervalo de medição de todas elas é de 10 minutos, havendo registros de no mínimo um ano (52.560 pontos) para cada série. A figura 9 mostra as séries temporais das curvas de vento de todos os sítios, onde o tempo está dado em minutos.

Foram calculadas as distribuições de frequência de ocorrência de velocidades de vento para as três séries utilizadas e posteriormente comparadas com uma distribuição de Weibull aproximada, conforme está representado na figura 10.

Conforme se pode visualizar na tabela 1 , que contém os dados estatísticos das séries temporais, o número de estados de velocidade de vento diferentes encontrado variou de 165 a 196, enquanto a velocidade média variou de 6,59 a 7,58 m/s.

Foram utilizadas as caracteristicas de uma turbina eólica real, aqui chamada Turbina A, cujos dados técnicos e a curva de potência estão dados na tabela 2 e figura 11, respectivamente.

As taxas de transição entre estados da turbina utilizada estão apresentadas na tabela 3 e foram sugeridas em (Sayas e Allan, 1996). Essas taxas foram utilizadas por não haver ainda no Brasil informações estatísticas que permitam calculá-las, dado o pouco tempo de operação e o reduzido número de usinas eólicas já implantadas no Brasil. Pela mesma razão, os índices calculados na seção seguinte serão os sugeridos na literatura internacional.
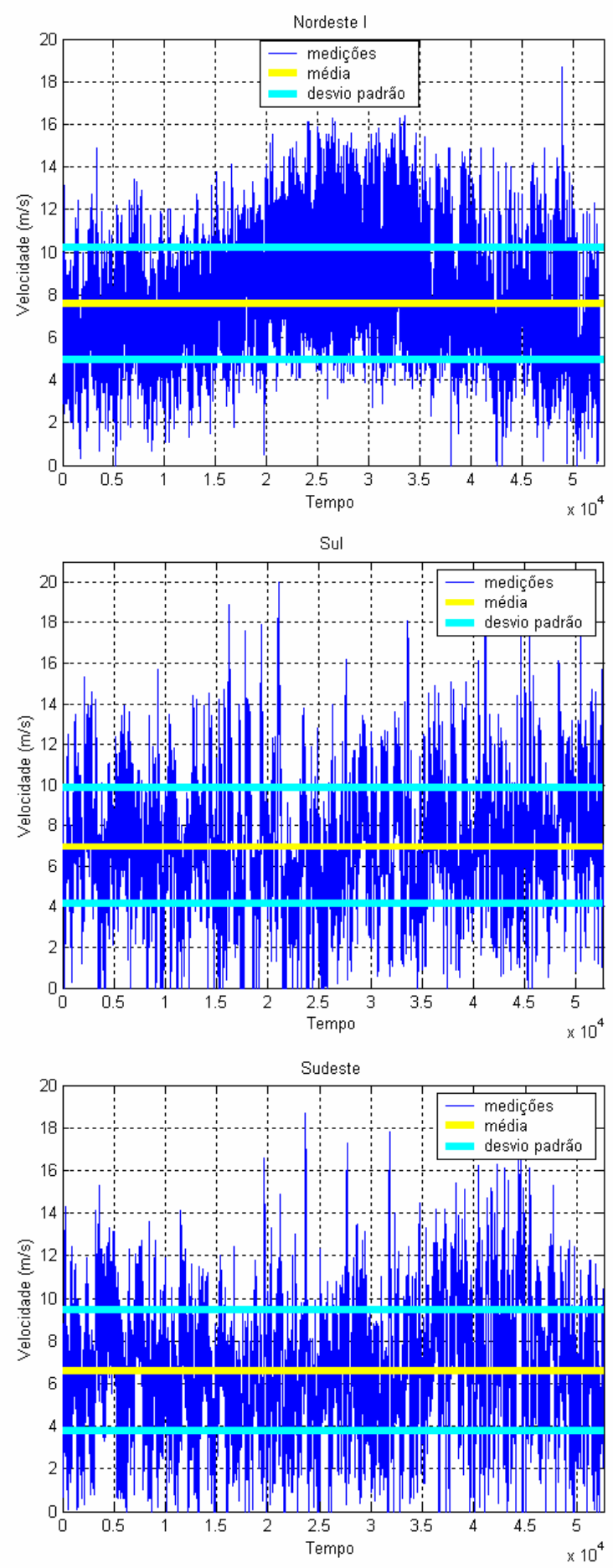

Figura 9: Séries temporais dos sítios estudados

\section{2 Índices Calculados}

Neste trabalho, o desempenho de usinas eólicas foi medido através de índices de confiabilidade definidos em (Allan e Billinton, 2000) e selecionados com o objetivo de avaliar 

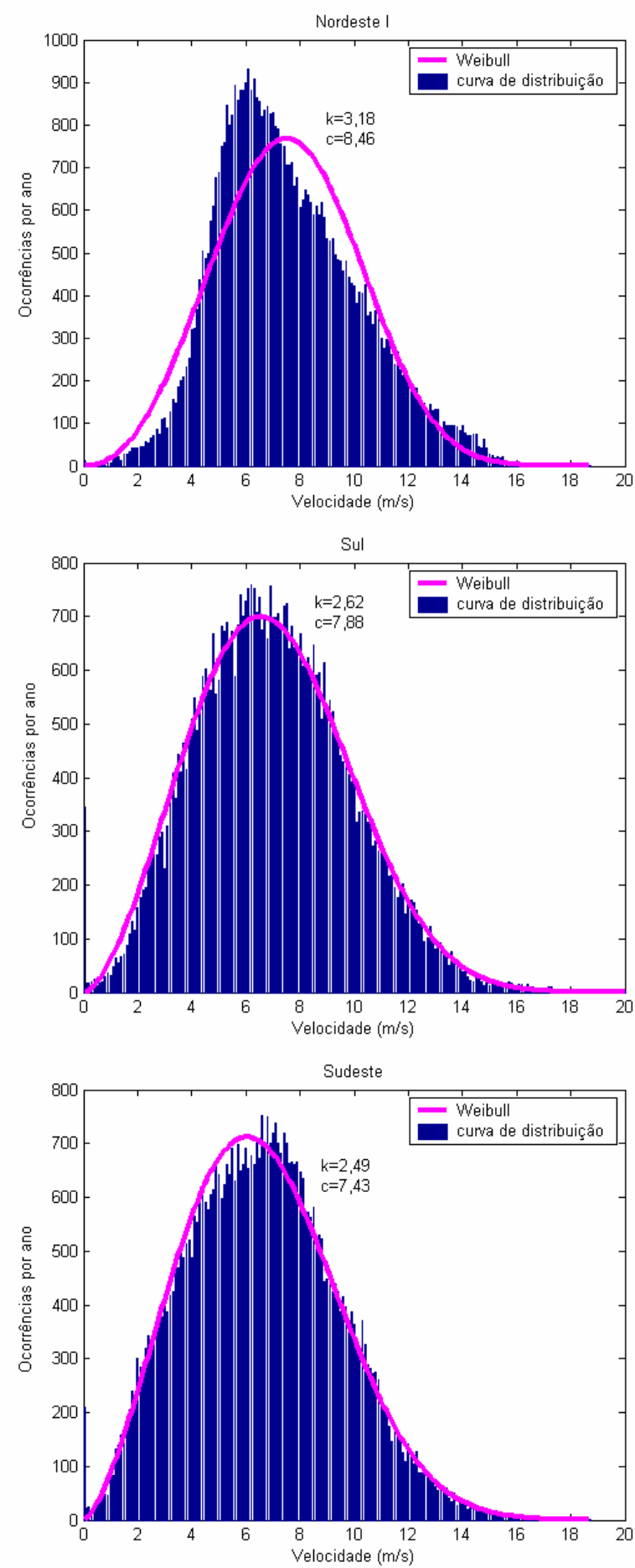

Figura 10: Distribuição de frequencia e de Weibull dos sítios

a disponibilidade de geração, a partir da disponibilidade de vento e da operação das turbinas da usina, a saber:

IWP (Installed Wind Power) - potência eólica instalada. É

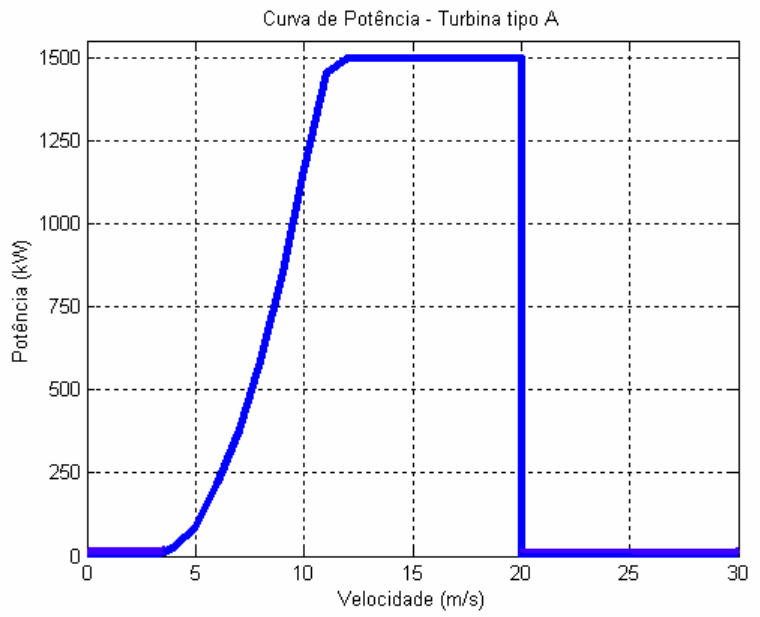

Figura 11: Curva de potência da turbina eólica

\begin{tabular}{|c|c|}
\hline $\begin{array}{c}\text { Taxa falha } \\
\text { (ocorrências/ano) }\end{array}$ & $\begin{array}{c}\text { Taxa reparo } \\
\text { (ocorrências/ano) }\end{array}$ \\
\hline 4 & 90 \\
\hline
\end{tabular}

Tabela 3: Taxas de falha e reparo utilizadas para a turbina

a soma das potências nominais de todas as turbinas da usina eólica. Unidade: [MW]

IWE (Installed Wind Energy) - potência eólica instalada multiplicada por pelo número de horas em um ano. Ela representa a energia máxima que pode ser extraída em um ano. Unidade: [MWh]

$$
I W E=I W P \times 8760
$$

EAWE (Expected Available Wind Energy) - expectativa de energia eólica disponível no vento. Quantidade de energia que pode ser gerada em um ano sem considerar saídas de turbinas eólicas. Unidade: [MWh]

EGWE (Expected Generated Wind Energy) - expectativa de energia eólica gerada. Quantidade de energia que pode ser gerada em um ano, considerando-se as saídas forçadas das turbinas. Unidade: [MWh]

WGAF (Wind generation availability factor) - fator de disponibilidade de geração eólica. Equivale ao fator de carga de usinas convencionais, mas considera-se também o efeito de operação das turbinas. Adimensional.

$$
W G A F=\frac{E G W E}{I W E}
$$




\begin{tabular}{|c|c|c|c|c|}
\hline \multirow{2}{*}{ Índice } & \multirow{2}{*}{ Unidade } & \multicolumn{3}{|c|}{ Número de Agrupamentos } \\
\cline { 3 - 5 } & & 10 & 80 & 165 \\
\hline EAWE & $\mathrm{kWh}$ & 5.103 .004 & 5.070 .784 & 5.069 .084 \\
\hline EGWE & $\mathrm{kWh}$ & 4.811 .659 & 4.821 .797 & 4.850 .817 \\
\hline WGAF & - & 0,3662 & 0,3670 & 0,3692 \\
\hline
\end{tabular}

Tabela 4: Índices de desempenho para vários agrupamentos

Além destes, pode-se utilizar o fator de capacidade $F C$ da usina eólica, que não considera o efeito da operação da turbina, apenas a disponibilidade de vento:

$$
F C=\frac{E A W E}{I W E}
$$

\subsection{Influência do Agrupamento do Vento}

O desempenho do modelo foi testado primeiramente com a série temporal do sítio Nordeste para 10, 80 e 165 agrupamentos de estados de velocidade de vento. Foi utilizada uma usina com apenas uma turbina.

A potência gerada para cada um dos estados de vento é obtida através da curva de potência da turbina, conforme mostra a figura 12 , onde estão representados também os valores de geração correspondentes a cada estado de vento.

A figura 13 mostra as curvas de duração, para as três condições de agrupamento analisadas, dentre outras. Pode-se notar que a representação do vento em poucos estados provocou uma estimativa otimista da geração, e somente a partir de 80 estados foi obtida uma representação próxima àquela com 165 estados.

A tabela 4 contém os índices de desempenho da usina, para todos os agrupamentos de velocidade analisados. O IWP, que representa a potência instalada, é $1.500 \mathrm{~kW}$ e o valor do IWE é de 13,14 GWh em todas as situações analisadas. Observase que a variação dos índices com o número de estados representados foi pequena.

As probabilidades de geração da usina podem ser separadas em quatro principais grupos:

- Probabilidade de geração não nula, com a velocidade de vento dentro de seus limites de acionamento e corte e pelo menos uma das turbinas da usina em operação.

- Probabilidade de não gerar por restrição de vento, ou seja, a geração da usina é zero porque o vento está fora de seus limites de acionamento ou de corte.

\begin{tabular}{|l|c|c|c|}
\hline \multirow{2}{*}{ Probabilidades (\%) } & \multicolumn{3}{|c|}{ Número de estados } \\
\cline { 2 - 4 } & 10 & 80 & 165 \\
\hline Geração & 92,489 & 93,262 & 93,799 \\
\hline Não gera - restrição de vento & 1,919 & 1,935 & 1,945 \\
\hline Não gera - restrição de turbina & 5,481 & 4,706 & 4,169 \\
\hline $\begin{array}{l}\text { Não gera - restrição de turbina } \\
\text { e vento }\end{array}$ & 0,111 & 0,096 & 0,086 \\
\hline
\end{tabular}

Tabela 5: Probabilidades de geração para vários agrupamentos

- Probabilidade de não gerar por restrição de turbina, ou seja, a geração da usina é zero porque todas as turbinas estão em estado de falha.

- Probabilidade de não gerar por restrição de turbina e de vento, ou seja, as duas possibilidades anteriores ocorrem ao mesmo tempo.

A tabela 5 representa essas probabilidades para os agrupamentos de velocidade analisados. Pode-se observar que a probabilidade da usina estar gerando cresceu com o aumento do número de agrupamentos, enquanto a probabilidade de não haver geração por restrição de turbina diminuiu. A probabilidade de não haver geração por restrição de velocidade de vento manteve-se praticamente constante para todos os agrupamentos analisados.

O número ideal de agrupamentos de estados de velocidade dependerá das características do regime de vento local. Neste caso, um agrupamento em 80 patamares, que corresponde a aproximadamente metade das velocidades diferentes encontradas no sítio, representou adequadamente o comportamento do vento. Por outro lado, verificou-se que a variação dos resultados encontrados para diferentes agrupamentos foi pequena. Portanto, conclui-se que o número de estados escolhido para o agrupamento da velocidade dependerá da precisão que se deseja obter e da capacidade computacional disponível, já que as séries temporais possuem um número de estados de vento muito grande, o que transforma a matriz de intensidade de transição em uma matriz de proporções elevadas.

\subsection{Influência do Número de Turbinas}

Considerando-se ainda o sítio Nordeste, explorou-se uma variação no número de turbinas para 10 e 20 unidades. A tabela 6 mostra os índices de desempenho calculados considerandose todos os estados de velocidade de vento deste sítio (165 estados) e a tabela 7 mostra as probabilidades de geração.

O aumento do número de turbinas não alterou o valor do ín- 

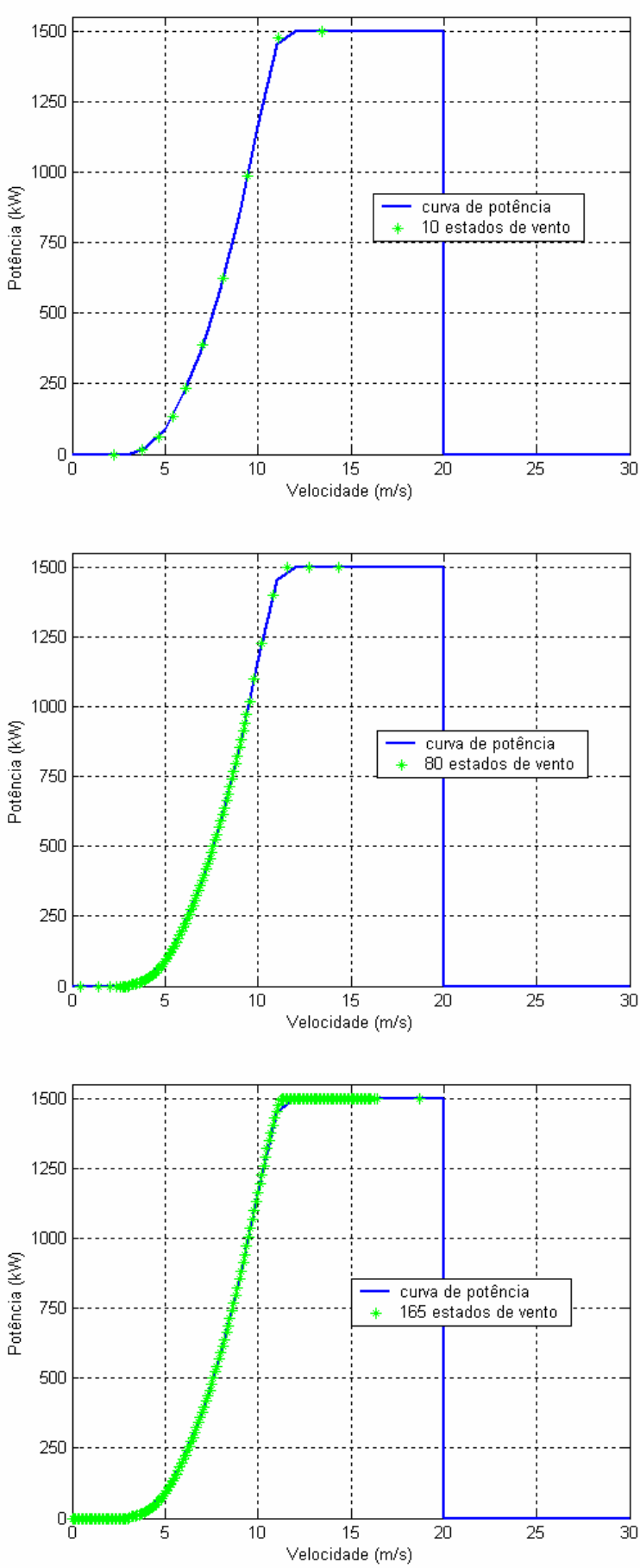

Figura 12: Representação dos estados de velocidade de vento na curva de potência para 10, 80 e 165 agrupamentos

dice de desempenho WGAF, mostrando que a proporcionalidade dos valores obtidos para os índices EAWE e EGWE foi mantida. Quanto às probabilidades de geração, verificouse que a probabilidade de não haver geração por restrição

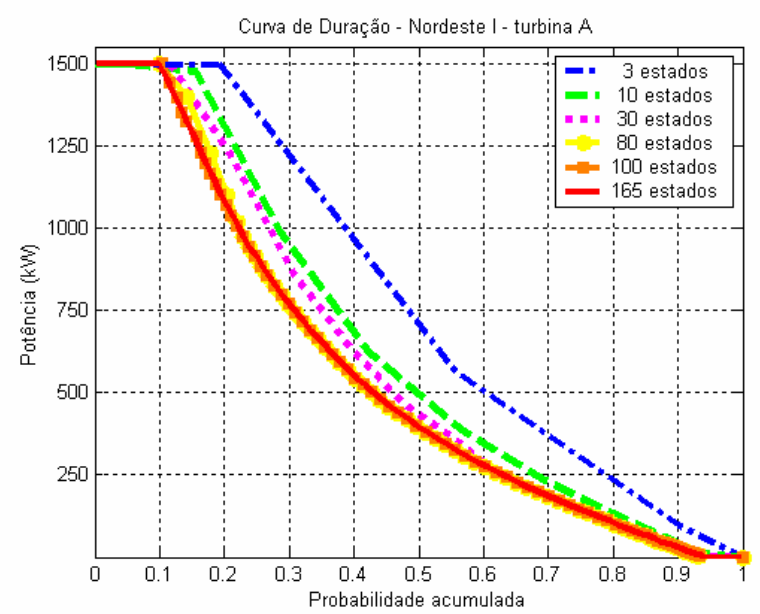

Figura 13: Curvas de Duração para diferentes agrupamentos

\begin{tabular}{|c|c|c|c|}
\hline \multirow{2}{*}{ Índice } & \multirow{2}{*}{ Unidade } & \multicolumn{2}{|c|}{ Número de turbinas } \\
\cline { 3 - 4 } & & 10 & 20 \\
\hline IWP & $\mathrm{kW}$ & 15.000 & 30.000 \\
\hline IWE & $\mathrm{kWh}$ & 131.395 .000 & 262.790 .000 \\
\hline EAWE & $\mathrm{kWh}$ & 50.690 .842 & 101.381 .684 \\
\hline EGWE & $\mathrm{kWh}$ & 48.508 .140 & 97.016 .080 \\
\hline WGAF & - & 0,3692 & 0,3692 \\
\hline
\end{tabular}

Tabela 6: Índices de desempenho para diferentes números de turbinas (165 estados de vento)

de turbina reduziu significativamente de valor quando houve aumento do número de turbinas, mostrando que as falhas das turbinas, em algumas situações, pode ser desprezada.

Foi analisada também a influência do número de agrupamentos de estados de velocidade para mais de uma turbina e verificou-se que a influência do número de agrupamentos é a mesma que ocorre quando somente uma turbina é analisada.

\subsection{Estimativa de Geração das Usinas}

A geração anual calculada pelo modelo desenvolvido foi comparada com a estimativa da geração convencional, baseada na distribuição de Weibull, utilizando-se em todos os sítios a Turbina A. Os resultados estão mostrados na tabela 8 .

É importante lembrar que, para o modelo proposto neste trabalho, a geração anual e o fator de capacidade correspondem aos índices EGWE e WGAF, mas isto não é verdade para a estimativa convencional, que considera apenas a variação na velocidade de vento e pressupõe que as turbinas estão dispo- 


\begin{tabular}{|l|c|c|}
\hline \multirow{2}{*}{ Probabilidades (\%) } & \multicolumn{2}{|c|}{ Número de turbinas } \\
\cline { 2 - 3 } & 10 & 20 \\
\hline Geração & 97,968 & 97,968 \\
\hline Não gera - restrição de vento & 2,032 & 2,032 \\
\hline Não gera - restrição de turbina & $4,658 \mathrm{E}-12$ & $1,948 \mathrm{E}-12$ \\
\hline $\begin{array}{l}\text { Não gera - restrição de turbina } \\
\text { e vento }\end{array}$ & $5,770 \mathrm{E}-14$ & $5,405 \mathrm{E}-15$ \\
\hline
\end{tabular}

Tabela 7: Probabilidades de geração para diferentes números de turbinas (165 estados de vento)

\begin{tabular}{|l|c|c|c|}
\hline $\begin{array}{c}\text { Estimativa de } \\
\text { Geração }\end{array}$ & Nordeste & Sul & Sudeste \\
\hline \multicolumn{3}{|c|}{ Estimativa Convencional (Weibull) } \\
\hline $\begin{array}{l}\text { Energia gerada } \\
\text { kWh/ano) }\end{array}$ & 5.249 .926 & 4.457 .407 & 3.903 .060 \\
\hline $\begin{array}{l}\text { Fator de } \\
\text { capacidade FC }\end{array}$ & 0,3995 & 0,3392 & 0,2970 \\
\hline \multicolumn{3}{|c|}{ Calculado pelo Modelo Proposto } \\
\hline $\begin{array}{l}\text { EAWE } \\
\text { kWh/ano) }\end{array}$ & 5.069 .084 & 4.432 .528 & 3.944 .223 \\
\hline $\begin{array}{l}\text { EGWE } \\
\text { (kWh/ano) }\end{array}$ & 4.850 .817 & 4.242 .601 & 3.772 .691 \\
\hline $\begin{array}{l}\text { Fator de } \\
\text { capacidade FC }\end{array}$ & 0,3858 & 0,3373 & 0,3002 \\
\hline WGAF & 0,3692 & 0,32288 & 0,28711 \\
\hline
\end{tabular}

Tabela 8: Estimativa de geração pelo modelo convencional e pelo modelo proposto para todos os sítios estudados

níveis para geração todo o tempo. Por essa razão, ela será sempre otimista em relação ao modelo desenvolvido. Dessa forma, a energia gerada, calculada utilizando-se a distribuição de Weibull, tem um valor mais elevado que o esperado na realidade e calculado pelo modelo proposto.

\subsection{Desempenho Computacional}

A estimativa do esforço computacional foi feita utilizando-se um computador Pentium 4 - 2,66 GHz. O tempo de simulação variou de $86 \mathrm{seg}$, para 1 turbina e 10 agrupamentos de estados, até $20 \mathrm{~min}$, para 20 turbinas e todos os estados de vento representados. O tempo de simulação para os diferentes agrupamentos de velocidades de vento e diferentes quantidades de turbinas mostrou que, nas situações onde poucas turbinas são representadas, a utilização de todos os estados de velocidade de vento é indicada. Dependendo das características do vento, para uma usina com muitas turbinas, podese optar pela utilização de um agrupamento com menor nú- mero de velocidades, para se obter um tempo de simulação razoável.

\section{CONCLUSÕES}

A crescente utilização da geração eólica requer modificações na concepção de planejamento de sistemas elétricos, pois inclui mais uma componente de incerteza, que precisa ser devidamente estudada e modelada. Entretanto, muitos trabalhos que avaliam o impacto da geração eólica na confiabilidade ainda representam as usinas eólicas como usinas convencionais. Como foi discutido neste trabalho, devido à forte característica intermitente do vento, uma modelagem probabilística que considere as suas especificidades deve ser utilizada. Este trabalho teve como objetivo o desenvolvimento de um modelo computacional de representação probabilística da geração de usinas eólicas para estudos de confiabilidade e de viabilidade de implantação.

Através dos resultados obtidos, confirmou-se a suposição de que em períodos de tempo relativamente longos (um ano ou mais), a velocidade do vento pode ser modelada por um processo de Markov a múltiplos estados. A modelagem probabilística da usina eólica mostrou-se útil em estudos de confiabilidade, pois foi capaz de fornecer os estados de geração e suas probabilidades de ocorrência, aliado a vários índices de desempenho. Além disso, a estimativa de geração e o fator de capacidade calculados pelo modelo consideram não só a variação da velocidade do vento, mas também as saídas forçadas das turbinas, produzindo um valor mais realista.

A estimativa da geração anual dos sítios estudados demonstrou as características favoráveis dos ventos à geração eólica no Brasil. Os fatores de capacidade calculados estiveram entre 30 e $40 \%$, valor considerado alto para os padrões mundiais. Além disso, constatou-se que a contribuição que as usinas eólicas podem fornecer a um sistema elétrico depende fortemente da combinação das turbinas usadas e do comportamento do vento local. Uma usina atrativa para uma empresa pode não ser para outra, dependendo da combinação escolhida.

\section{REFERÊNCIAS}

Allan, R., Billinton, R. (2000). Probabilistic Assessment of Power Systems. Proceedings of the IEEE, Vol. 88, No. 2, pp. 140-162.

Billinton, R., Chen, H., Chajar, R. (1996). A Sequential Simulation Technique for Adequacy Evaluation of Generating Systems Including Wind Energy. IEEE Transactions on Energy Conversion, Vol. 11, No. 4, pp. 728734. 
Billinton, R., Karki, R. (2001). Maintaining Supply Reliability of Small Isolated Power Systems Using Renewable Energy. IEE Proc.-Gener. Transm. Distrib., Vol. 148, No. 6, pp. 530-534.

Corotis, R. B., Sigi, A. B., Klein, J. (1978). Probability Models of Wind Velocity and Magnitude and Persistence. Solar Energy, Vol. 20, pp. 483-493, 1978.

Giorsetto, P., Utsurogi, K. F. (1983), Development of a New Procedure for Reliability Modeling of Wind Turbine Generators. IEEE Transactions on Power Apparatus and Systems, Vol. PAS-102, No. 1, pp 134-143.

MacQueen, J. (1967), Some Methods for Classification and Analysis of Multivariate Observation, Proceedings of the Fifth Berkeley Symposium on Mathematical Statistics and Probability, v. 1, pp. 281-297, University of California Press, Berkeley, 1967.

Puntar, S. G. (2003). Métodos e Visualização de Agrupamento de Dados. Tese de Mestrado, COPPE/UFRJ, Rio de Janeiro - RJ.

Sayas, F. C., Allan, R. N. (1996). Generation Availability Assessment of Wind Farms. IEE Proc.-Gener. Transm. Distrib., Vol. 143, No. 5.

Singh, C., Kim, Y. (1988). An Efficient Technique for Reliability Analysis of Power Systems Including Time Dependent Sources. IEEE Transactions on Power Systems, Vol. 3, No. 3, pp. 1090-1096.

Thomann, G. C., Barfield, M. J. (1998). The Time Variation of Wind Speeds and Windfarm Power Output in Kansas. IEEE Transactions on Energy Conversion, Vol. 3, No. 1, pp. 44-49. 